\title{
Composites
}

\section{The variance on the shock response of a carbon fibre composite due to the orientation of the weave}

\author{
D. C. Wood ${ }^{1, \star}$, G. J. Appleby-Thomas ${ }^{1}$, A. Hameed ${ }^{1}$, N. R. Barnes ${ }^{2}$, A. Hughes ${ }^{2}$, and P. J. Hazell ${ }^{3}$ \\ ${ }^{1}$ Centre for Defence Engineering, Cranfield University, Defence Academy of the UK, Shrivenham SN6 8LA, UK \\ ${ }^{2} A W E$, Aldermaston, Reading RG7 4PR, UK \\ ${ }^{3}$ School of Engineering and Information Technology, The University of New South Wales, UNSW Canberra, PO Box 7916, Canberra, \\ BC 2610, Australia
}

Received: 20 February 2018

Accepted: 9 May 2018

Published online:

22 May 2018

(C) The Author(s) 2018

\begin{abstract}
Three different orientations of a tape-wrapped carbon fibre composite with phenolic resin matrix (abbreviated to TWCP) have been investigated under onedimensional shock loading. This has been achieved via a single-stage gas gun, with manganin gauges as the diagnostic tool. The orientations of TWCP studied in this paper were $25^{\circ}, 45^{\circ}$ and $90^{\circ}$, with respect to the impact face. The shock response of these orientations, for this material, has been obtained (the Hugoniot equation of state). These results have been contrasted with previously reported literature data for the same material at different orientations $\left(0^{\circ}\right.$ and $\left.20^{\circ}\right)$. It was found that orientation had minimal effect on the behaviour of this composite under shock. The exception to this was the $90^{\circ}$ orientation which exhibited an elastic precursor at particle velocities of less than $0.65 \mathrm{~mm} \mathrm{\mu s}^{-1}$; where the shock velocity was equivalent to the elastic sound speed of the material.
\end{abstract}

\section{Introduction}

Composites have a high strength to weight ratio making them ideal candidates for multiple applications in a wide range of industries, for example the aerospace industry [1-3]. Due to these numerous industries and therefore applications, a wide range of conditions may be experienced such as shock loading.

When looking at the shock response of composite materials, a multitude of different configurations can alter the observed behaviour. These can include constituents used (such as fibre ratio, type of fibre or matrix) [4, 5], configuration of the layered structure [6], thickness of the composite sample [7, 8] and the orientation of constituent layer [9-11] which is the focus of this paper.

To understand the behaviour of shocked materials, five parameters are needed. These parameters are shock velocity $U_{\mathrm{S}}$, particle velocity $u_{\mathrm{p}}$, density $\rho$, pressure $P$ and internal energy $E$. With any two of these five parameters obtained, the materials shock response can be fully described using the RankineHugoniot equations [12] (which are based on principles of conservation of mass, momentum and energy). The two parameters directly measured in

Address correspondence to E-mail: d.wood@cranfield.ac.uk 
this study were $U_{\mathrm{S}}$ and $\sigma_{x}$, with a third parameter $u_{\mathrm{p}}$ found using the impedance matching technique [12]. Further to these parameters, density was calculated from the Rankine-Hugoniot jump equations (with the inverse of density giving the volume, $V$ ). This allowed the Hugoniot in both the $U_{\mathrm{S}}-u_{\mathrm{p}}$ and pressure-volume planes to be ascertained.

In the $U_{\mathrm{S}}-u_{\mathrm{p}}$ plane, the Hugoniot relationship tends to be linear in nature for most materials, following the form shown in Eq. (1). Nonlinear behaviour in the $U_{\mathrm{S}}-u_{\mathrm{p}}$ Hugoniot plane has been observed with some materials such as polymers [13-15], including the matrix material of TWCP investigated here [16]. For Eq. (1), $c_{0}$ is the Hugoniot intercept at the ground state which tends to be comparable to $\mathcal{c}_{\mathrm{B}}$ (the bulk sound velocity of the material, given by Eq. 5); while $S$ is the slope of the Hugoniot.

$U_{\mathrm{S}}=c_{0}+S u_{\mathrm{p}}$

The hydrodynamic pressure of a material $\left(P_{\mathrm{H}}\right.$, also referred to as the Hugoniot pressure) is given by Eq. (2) and is typically represented in the pressureparticle velocity plane. This equation may be adjusted for use in the pressure-volume plane with this form given in Eq. (3), where $\rho_{0}$ is the initial density and $V$ along with $V_{0}$ are the volume at the Hugoniot pressure and initial state, respectively.

$P_{\mathrm{H}}=\rho_{0} U_{\mathrm{S}} u_{\mathrm{p}}$

$P_{\mathrm{H}}=\rho_{0} U_{\mathrm{S}}^{2}\left(1-\frac{V}{V_{0}}\right)$

The Hugoniot pressure and deviatoric (maximum shear stress $\tau$, derived from the Cauchy stress tensor [17]) elements of stress are linked by Eq. (4). This relation implies that if the longitudinal stress equals the hydrodynamic pressure then there will be little to no strength effect in the material.

$\sigma_{x}=P_{\mathrm{H}}+\frac{4}{3} \tau$

Due to the number of different constituents and arrangements, a wide range of composite systems are commercially available. This adjustment in the composite material due to a change in constituents can greatly alter the material response especially in the shock regime. This adjustment could be a change in fibres, matrix or of the volume fraction of the material, for example. For the purposes of this investigation, the property under consideration was the orientation of the fibre weave with respect to the shock front in a carbon fibre composite.

Many authors have investigated the effect that orientation has on the shock response of composites; however, they tend to focus on the orientations parallel and perpendicular to the shock front, a good overview on the shock response of composites is given by Ref. [18].

One study that investigated a wide range of orientations and its affect on the shock profile was the work of Bordzilovsky et al. [10]. In this paper, an aramid fibre composite with an epoxy resin matrix material was investigated. A single set of experimental conditions was picked, utilising TNT to impart a shock into the sample, such that any difference in the shock profile was due to the orientation of the aramid fibre weave. The angles of the fibre weave investigated were $5^{\circ}, 15^{\circ}, 45^{\circ}$ and $90^{\circ}$, with respect to the direction of shock wave. An elastic precursor was noted at the orientations of $5^{\circ}$ and $15^{\circ}$ before finally smearing and disappearing at $90^{\circ}$.

Millett et al. [9] investigated the effect that orientation of a carbon fibre weave had on the resultant shock profile. In this study, two orientations of fibre weaves were investigated; one where the fibre weave was orientated parallel to the shock front, with the other fibre weave orientated perpendicular to the shock front. To impart the shock wave, aluminium or copper flyers were used at velocities between 200 and $1125 \mathrm{~m} \mathrm{~s}^{-1}$. It was found that when the fibre weave was parallel to the shock front the carbon fibre composite behaved more like a monolithic material; with the exception of very small oscillations on the plateau of the trace. These were attributed to shock wave interactions between the fibres and matrix within the sample material. However, when the fibre weave was orientated perpendicular to the shock front, a ramped elastic portion was seen with an approximate velocity of $7 \mathrm{~mm} \mu \mathrm{s}^{-1}$, before the main shock front overtook the elastic portion. This behaviour was explained as an elastic wave travelling down the fibres ahead of the main shock front. Despite this behaviour, both Hugoniots in the $U_{\mathrm{S}}-u_{\mathrm{p}}$ plane were found to be linear in nature, with significant deviation at lower particle velocities before convergence was observed at elevated particle velocities.

Hazell et al. [7] also investigated a carbon fibre composite, where the weave was orientated perpendicular to the shock front. The main focus of this 
paper was the effect of thickness on the shock response of this composite. The four thicknesses used were 1.52, 3.00, 6.05 and $9.06 \mathrm{~mm}$. As observed in the study by Millett et al. [9], an initial ramped portion due to an elastic precursor through the fibres was seen. As the thickness of the composite was increased, the initial ramped portion of the trace became more pronounced, as did the magnitude of pressure of this initial ramped portion.

Multiple diagnostic techniques were used by Willows et al. [19] to investigate the shock response of a carbon fibre composite using the plate impact technique. By employing both VISAR and manganin stress gauges, on ring up and ring down experiments the shock profile was investigated. It was concluded, via the use of a computational model that fibre orientation was unimportant above a particle velocity value of approximately $1000 \mathrm{~m} \mathrm{~s}^{-1}$. This supports the work conducted by Millett et al. [9], who observed Hugoniot convergence with their composite at higher particle velocities.

In this study, a tape-wrapped carbon fibre composite with a phenolic resin (abbreviated to TWCP) will be investigated. This material has been studied previously by Wood et al. [20] and Burrell et al. [1] at orientations of $0^{\circ}$ and $20^{\circ}$, respectively. These orientations were with respect to the impact surface of the sample.

Wood et al. [20] investigated a TWCP composite where the fibre weave was arranged parallel to the shock front. As was observed by Millett et al. [9] on their carbon fibre composite, the $0^{\circ}$ weave orientation behaved monolithically, with the exception of noted oscillations on the plateau of the experimental gauge traces. These oscillations were found to be due to the thickness of the fibre weave and the shock interactions between the carbon fibre weave and the matrix material. It was noted in this study that convergence between the TWCP and the phenolic resin matrix material occurred above a particle velocity of

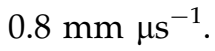

The shock response of a TWCP composite, with the weave orientated at $20^{\circ}$ to the impact face was investigated by Burrell et al. [1]. Unlike the one investigated by Wood et al. [20] and in this study, the TWCP material investigated by Burrell et al. had a different compositional makeup, as noted by the difference in the initial density. The shock response was compared to previously obtained data for TWCP, where good agreement between the data sets was noted. Within the data, there was no indication of any elastic behaviour, as noted in composites studied by Bordzilovsky et al. [10], Millett et al. [9] and Hazell et al. [7].

In this paper, the previous work conducted by Wood et al. [20] and Burrell et al. [1] will be built upon and expanded to include more orientations. The orientations that will be investigated here are $25^{\circ}$, $45^{\circ}$ and $90^{\circ}$ with the orientation referring to the angle between the shock front and the fibre weave. These data will then be compared to previous work in the literature (specifically Refs. [1, 20]), to see the effect that orientation has on the shock response of this TWCP material.

\section{Experimental technique}

The plate impact technique was used to induce a onedimensional shock wave into the TWCP samples. To achieve this, a single-stage gas gun with a 5-m-long barrel and 50-mm bore was used to accelerate flyer plates (attached to sabots for stability) into the targets. The flyer plates used in these experiments were aluminium or copper (either 5 or $10 \mathrm{~mm}$ thick) due to their well characterised behaviour while under shock [21]. Velocity measurements were obtained via a series of sequential infrared transmitters and receivers. This experimental setup is shown simplistically in Fig. 1. One-dimensionality is assured by careful machining of all components within the targets such that all parallel surfaces to the shock front are flat and parallel to tolerances of $\pm 10 \mu \mathrm{m}$. To confirm the accuracy of the gas gun with relation to the target, tilt pins experiments are performed before and after shot programs. The maximum misalignment of the coverplate surface to flyerplate surface within this program of work was found to be $1.9 \mathrm{mrad}$.

To investigate the effect that orientation has on the shock response of composites manganin pressure gauges were used to monitor the transit of the shock profile. Manganin is used as it is largely unaffected by the temperature increase associated with a shock wave, and as such the output is the pressure of the material (assuming hydrodynamic behaviour). The gauges employed within these experiments were manufactured by Vishay Micro-measurements with the product designation being LM-SS-125CH-048, with an active manganin grid element of 3.18 by $4.45 \mathrm{~mm}$ for a total active area of $14.15 \mathrm{~mm}^{2}$. When a 


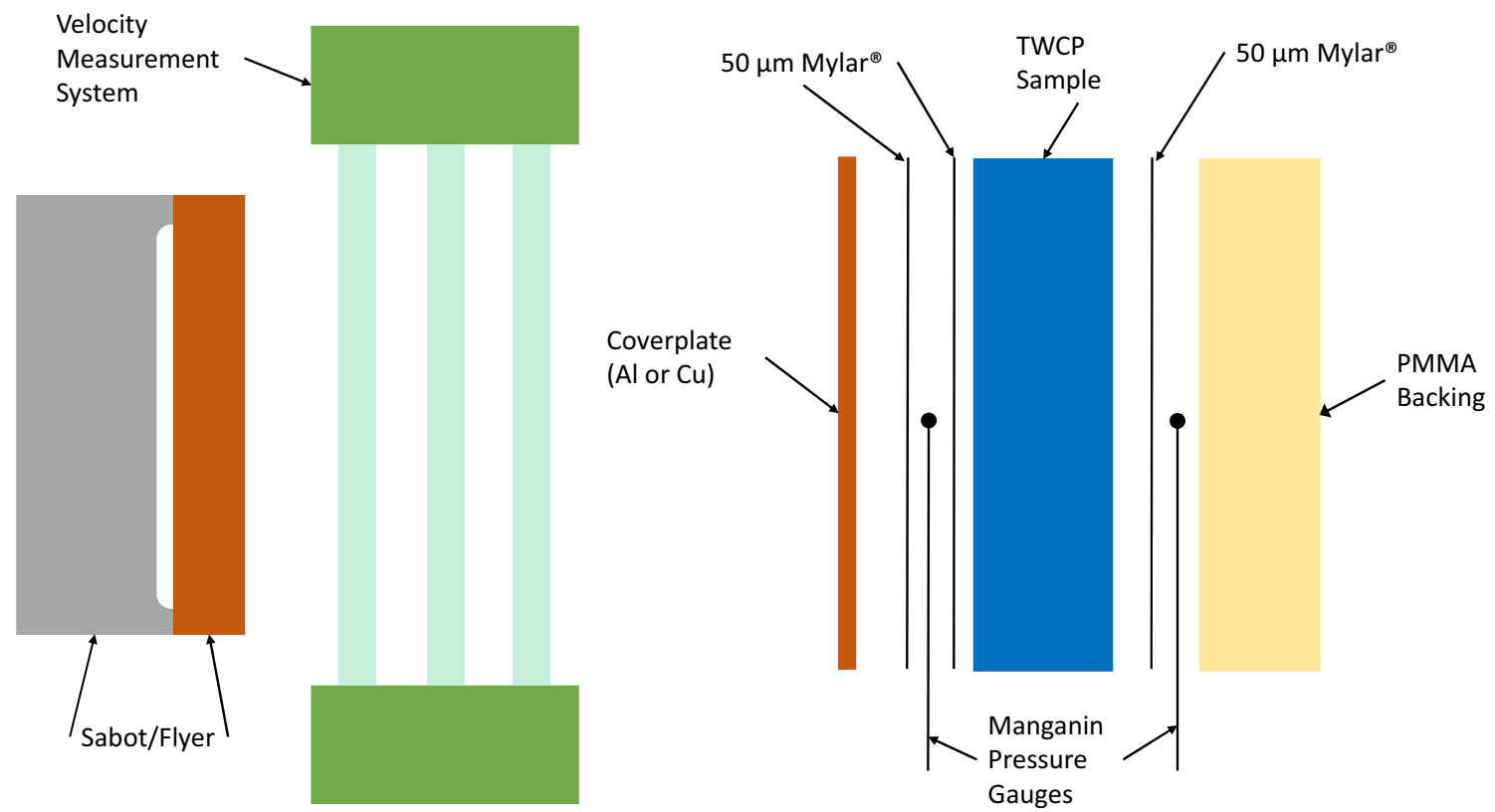

Figure 1 General experimental setup, with typical target configuration.

pressure is applied to the gauge a proportional change in resistance is noted, which by using the calibration method of Rosenberg et al. [22] can be calculated.

Figure 1 shows a simplified target setup arrangement. One thing to note is the inclusion of $50 \mu \mathrm{m}$ of Mylar ${ }^{\circledR}$ which is used as both electrical and physical protection of the gauges. All matching surfaces of the components were joined together with a slow curing epoxy resin Locite $0151 \mathrm{HYSOL}^{\mathrm{TM}}$ Epoxi-Patch $^{\circledR}$. In these experiments, all samples were of dimensions 60 by 60 by $8 \mathrm{~mm}$.

\section{Material properties}

For the material investigated here, the carbon fibres are woven into a two-dimensional fabric (plain weave), which are stacked upon one another, before being impregnated by the phenolic resin (Durite SC1008 previously investigated by Wood et al. [16]). It is these weaves that are orientated with respect to the shock front. Density measurements were conducted using a Micrometrics AccuPyc 1330 gas pycnometer which gave a value of $1.46 \mathrm{~g} \mathrm{~cm}^{-3}$ for all of the orientations of TWCP. The orientation investigated by Burrell et al. [1] had a slightly higher density at $1.48 \mathrm{~g} \mathrm{~cm}^{-3}$ due to manufacturing techniques, hence the discrepancy between the densities in Table 1. For all orientations of this TWCP material, ultrasonic measurements have been obtained using $1 \mathrm{MHz}$ quartz transducers and a Panametrics 5077PR pulse receiver. Pulse-echo mode was used to obtain longitudinal sound speed (cL), with the shear sound speed (cS) found using transmit-receive configuration, with the bulk sound speed found via Eq. (5). Table 1 shows the key elastic properties for TWCP, $c_{L}, c_{S}, c_{B}$ and $v$ (Poisson's ratio) for all of the orientations investigated here, as well as previous work by Burrell et al. [1] and Wood et al. [20]. Poisson's ratio is given by Eq. (6) and is based on the sound speed of the materials, which leads to the discrepancy in Table 1 caused by the anisotropy of the composite.

$c_{\mathrm{B}}=\sqrt{c_{\mathrm{L}}^{2}-\frac{4}{3} c_{\mathrm{S}}^{2}}$

$v=\frac{0.5-\left(\frac{c_{\mathrm{S}}}{c_{\mathrm{L}}}\right)^{2}}{1.0-\left(\frac{c_{\mathrm{S}}}{c_{\mathrm{L}}}\right)^{2}}$

Using micrographs, the volume fraction of the TWCP composite was calculated as previously conducted in the work by Wood et al. [20]. This technique led to a volume fraction of $54 \pm 4 \%$ for all the orientations investigated in this study, with the fibre weave being between 300 and $600 \mu \mathrm{m}$. 
Table 1 Key elastic properties of TWCP for all of the orientations

\begin{tabular}{llllll}
\hline Cloth angle $\left(^{\circ}\right)$ & $\rho_{0}\left(\mathrm{~g} \mathrm{~cm}^{-3}\right)$ & $c_{\mathrm{L}}\left(\mathrm{mm} \mathrm{\mu s}^{-1}\right)$ & $c_{\mathrm{S}}\left(\mathrm{mm} \mathrm{\mu s}^{-1}\right)$ & $c_{\mathrm{B}}\left(\mathrm{mm} \mathrm{\mu s}^{-1}\right)$ & $v$ \\
\hline $0[20]$ & $1.46 \pm 0.01$ & $3.61 \pm 0.02$ & $2.00 \pm 0.02$ & $2.78 \pm 0.03$ & 0.28 \\
$20[1]$ & $1.48 \pm 0.01$ & $3.63 \pm 0.03$ & $0.99 \pm 0.03$ & $3.45 \pm 0.04$ & 0.46 \\
25 & $1.46 \pm 0.01$ & $3.55 \pm 0.02$ & $1.00 \pm 0.02$ & $3.36 \pm 0.03$ & 0.46 \\
45 & $1.46 \pm 0.01$ & $3.47 \pm 0.02$ & $1.04 \pm 0.02$ & $3.26 \pm 0.03$ & 0.45 \\
90 & $1.46 \pm 0.01$ & $4.20 \pm 0.02$ & $2.01 \pm 0.02$ & $3.50 \pm 0.03$ & 0.35 \\
\hline
\end{tabular}

With the manganin gauge diagnostic, an average shock response of the composite would be obtained. This is due to the dimensions of the gauge (mentioned in "Experimental technique" section) when compared to the dimensions of the fibre weave. For the worst case scenario of a weave thickness of $600 \mu \mathrm{m}$ there are between 5.3 and 7.4 layers across the active grid element depending on orientation with respect to each other, with the best case scenario being 10.6-14.8 layers across the active manganin gauge (for a $300 \mu \mathrm{m}$ fibre weave). Because of the averaging effect of the gauge, the shock response of the composite behaves more like a isotropic material, this behaviour noted previously by Hazell et al. [7] and Millett et al. [9]. As such, isotropic equations have been employed on this anisotropic material due to the relative size of the active gauge element and the composite layers. With a diagnostic that has higher spatial resolution such as Het-V or VISAR, which monitors over a potentially very small area, issues may arise, as it would be possible to focus on either the fibres/weave or the matrix independently.

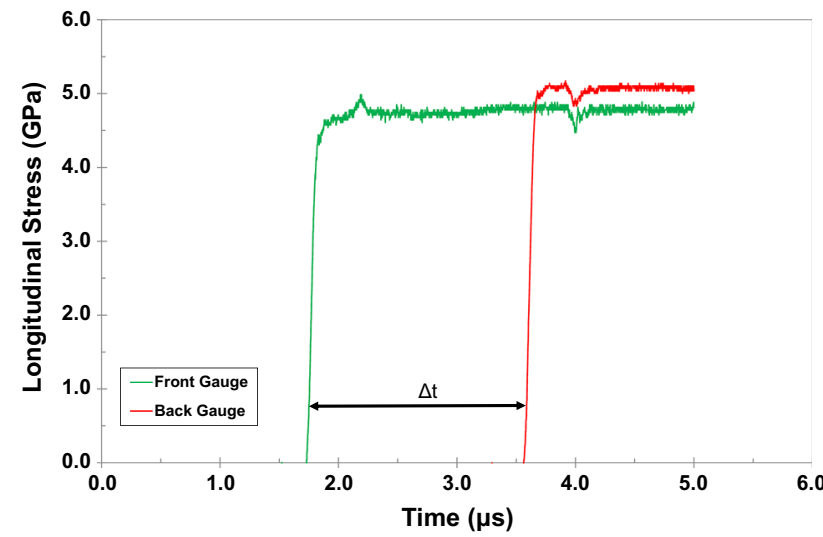

Figure 2 Typical gauge for the $90^{\circ}$ TWCP orientation where no elastic precursor behaviour was observed. Experimental conditions employed a $10 \mathrm{~mm} \mathrm{Cu}$ flyer impacting at $890 \mathrm{~m} \mathrm{~s}^{-1}$.

\section{Results and discussion}

Typical experimental data traces for the $90^{\circ}$ TWCP orientation are shown in Figs. 2 and 3. Typical shock traces such as the one shown in Fig. 2 follow the same basic form, with subtleties dependent on the material being investigated. Initially a sharp rise is seen on both the front and rear gauge traces (assuming no precursor wave was seen, as observed in Fig. 3) on the order of $70 \mathrm{~ns}$ for this experiment, with this value due to the thicker than normal insulation/protection layer $\left(50 \mu \mathrm{m}\right.$ instead of $25 \mu \mathrm{m}$ of Mylar $\left.^{\circledR}\right)$ being employed. After this rise, a plateau of $3 \mu$ s on the front gauge is present, and $1.5 \mu$ s on the rear gauge, which corresponds to the longitudinal stress observed by the gauge. After this plateau gauge death is seen, where no more information from the experiment is attainable.

From Fig. 2, with the experimental setup employed (shown in Fig. 1) the stress and shock velocity can be directly ascertained, via use of the manganin gauges. To calculate the value of $U_{S}$ the time ( $\Delta t$ in Fig. 2 ) is used with the known thickness of the sample and Mylar used. Shock velocity is simply then the

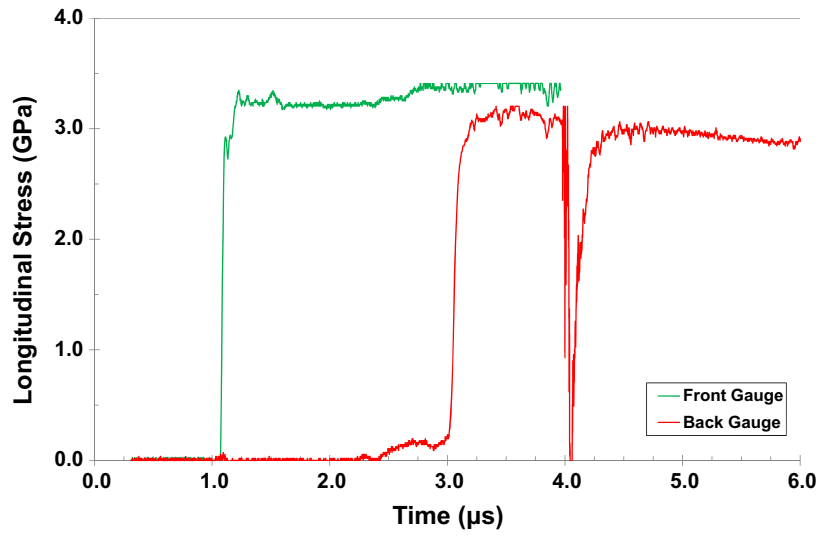

Figure 3 A representative trace for the $90^{\circ}$ TWCP orientation displaying the elastic precursor. Impact conditions were a $10 \mathrm{~mm}$ $\mathrm{Cu}$ flyer travelling at a velocity of $600 \mathrm{~m} \mathrm{~s}^{-1}$. 
distance between the gauges, divided by the time it takes the shock wave to interact with each gauge $(\Delta t)$.

Stress is obtained from the gauges after the raw data are interpreted according to the calibration by Rosenberg et al. [22]. Due to the difference in impedance between the target and PMMA backing plate the rear gauge pressure is adjusted according to Eq. (7). The rear gauge pressure would be the one associated with the target and not the PMMA backing material [23] as given by the current experimental setup would give with no adjustment.

$\sigma_{\text {Sample }}=\frac{1}{2} \frac{Z_{\text {Sample }}+Z_{\text {Backing }}}{Z_{\text {Backing }}} \sigma_{\text {Rear Gauge }}$

Particle velocity for these experiments is obtained from the impedance match technique described by Meyers [12]. This allow the experimental data to be calculated (as shown in Tables 2, 3, 4, 5), the Hugoniots in the $U_{\mathrm{S}}-u_{\mathrm{p}}$ and pressure-unitless $V$ plane (Figs. 4, 6, 8, 9, 10) allow the equation of state for each angle according to Eq. (1) to be ascertained (Eqs. 8, 9, 10, as well as collated together in Table 6).

Precursor waves were seen with the $90^{\circ}$ TWCP orientation, as seen in Fig. 3. It should be noted that the drop seen on the rear gauge is due to crosstalk of the gauges and occurs at the death of the front gauge. Precursor wave behaviour has been noted in multiple composites including aramid fibre [10], Dyneema ${ }^{\circledR}$ [24], glass fibre [11] and carbon fibre [7, 9]. This behaviour on the composite investigated here was only observed on experiments below a certain particle velocity, and will be discussed later in more depth.

The experimental data for the $90^{\circ}$ TWCP weave orientation is collated and shown in Table 2 with corresponding impact conditions.

This is plotted graphically in Fig. 4 for both the Hugoniot in the $U_{\mathrm{s}}-u_{\mathrm{p}}$ and pressure-unitless volume plane. Included on this graph is data from the literature of other previously studied orientation of the

Table 2 Experimental results for the TWCP with the cloth angled perpendicular to the shock front

\begin{tabular}{|c|c|c|c|c|c|}
\hline Velocity $\left(\mathrm{m} \mathrm{s}^{-1}\right)$ & Flyer thickness/material (mm) & $U_{\mathrm{S}}\left(\mathrm{mm} \mu \mathrm{s}^{-1}\right)$ & $u_{\mathrm{p}}\left(\mathrm{mm} \mu \mathrm{s}^{-1}\right)$ & Volume $\left(\mathrm{cm}^{3} \mathrm{~g}^{-1}\right)$ & $\sigma_{x}(\mathrm{GPa})$ \\
\hline 355 & 10 aluminium & 4.17 & 0.24 & 0.64 & 1.43 \\
\hline 530 & 10 copper & 4.15 & 0.45 & 0.61 & 2.73 \\
\hline 600 & 10 copper & 4.13 & 0.51 & 0.60 & 3.21 \\
\hline 690 & 10 copper & 4.08 & 0.59 & 0.59 & 3.70 \\
\hline 835 & 10 copper & 4.31 & 0.71 & 0.57 & 4.05 \\
\hline 890 & 10 copper & 4.40 & 0.76 & 0.57 & 4.76 \\
\hline 1015 & 5 copper & 4.39 & 0.87 & 0.55 & 5.61 \\
\hline
\end{tabular}

Table 3 Experimental results for the elastic precursor

\begin{tabular}{|c|c|c|c|c|c|}
\hline Impact velocity $\left(\mathrm{m} \mathrm{s}^{-1}\right)$ & Flyer thickness/materials (mm) & $\Delta x(\mathrm{~mm})$ & $\Delta t(\mu \mathrm{s})$ & $U_{\mathrm{S}}^{\mathrm{F}} / c_{\mathrm{L}}^{F}\left(\mathrm{~mm} \mu \mathrm{s}^{-1}\right)$ & $u_{\mathrm{p}}\left(\mathrm{mm} \mu \mathrm{s}^{-1}\right)$ \\
\hline 530 & 10 copper & 8.00 & 1.27 & 6.30 & 0.42 \\
\hline 600 & 10 copper & 8.00 & 1.31 & 6.12 & 0.48 \\
\hline 690 & 10 copper & 7.98 & 1.66 & 4.80 & 0.58 \\
\hline
\end{tabular}

Table 4 Experimental results for the TWCP with the cloth angled $45^{\circ}$ to the shock front

\begin{tabular}{|c|c|c|c|c|c|}
\hline Velocity $\left(\mathrm{m} \mathrm{s}^{-1}\right)$ & Flyer thickness/material (mm) & $U_{\mathrm{S}}\left(\mathrm{mm} \mu \mathrm{s}^{-1}\right)$ & $u_{\mathrm{p}}\left(\mathrm{mm} \mu \mathrm{s}^{-1}\right)$ & Volume $\left(\mathrm{cm}^{3} \mathrm{~g}^{-1}\right)$ & $\sigma_{x}(\mathrm{GPa})$ \\
\hline 350 & 10 aluminium & 3.87 & 0.25 & 0.64 & 1.40 \\
\hline 540 & 10 aluminium & 3.78 & 0.39 & 0.61 & 2.11 \\
\hline 550 & 10 aluminium & 3.82 & 0.39 & 0.61 & 2.17 \\
\hline 675 & 10 copper & 4.03 & 0.58 & 0.59 & 3.42 \\
\hline 700 & 10 copper & 4.04 & 0.60 & 0.58 & 3.73 \\
\hline 840 & 10 copper & 4.34 & 0.72 & 0.57 & 4.51 \\
\hline 985 & 5 copper & 4.41 & 0.84 & 0.56 & 5.51 \\
\hline
\end{tabular}


Table 5 Experimental results for the TWCP with the cloth angled $25^{\circ}$ to the shock front

\begin{tabular}{|c|c|c|c|c|c|}
\hline Velocity $\left(\mathrm{m} \mathrm{s}^{-1}\right)$ & Flyer thickness/material (mm) & $U_{\mathrm{S}}\left(\mathrm{mm} \mu \mathrm{s}^{-1}\right)$ & $u_{\mathrm{p}}\left(\mathrm{mm} \mu \mathrm{s}^{-1}\right)$ & Volume $\left(\mathrm{cm}^{3} \mathrm{~g}^{-1}\right)$ & $\sigma_{x}(\mathrm{GPa})$ \\
\hline 330 & 10 aluminium & 3.80 & 0.23 & 0.64 & 1.30 \\
\hline 350 & 10 aluminium & 3.86 & 0.25 & 0.64 & 1.51 \\
\hline 400 & 10 aluminium & 3.54 & 0.29 & 0.63 & 1.62 \\
\hline 440 & 10 aluminium & 3.54 & 0.32 & 0.62 & 1.78 \\
\hline 510 & 10 copper & 3.89 & 0.44 & 0.61 & 2.60 \\
\hline 535 & 10 aluminium & 3.48 & 0.39 & 0.61 & 2.21 \\
\hline 600 & 10 copper & 3.93 & 0.52 & 0.59 & 3.08 \\
\hline 690 & 10 copper & 3.88 & 0.60 & 0.58 & 3.74 \\
\hline 820 & 10 copper & 3.74 & 0.71 & 0.55 & 4.36 \\
\hline 1155 & 5 copper & 4.34 & 0.99 & 0.53 & 6.79 \\
\hline
\end{tabular}
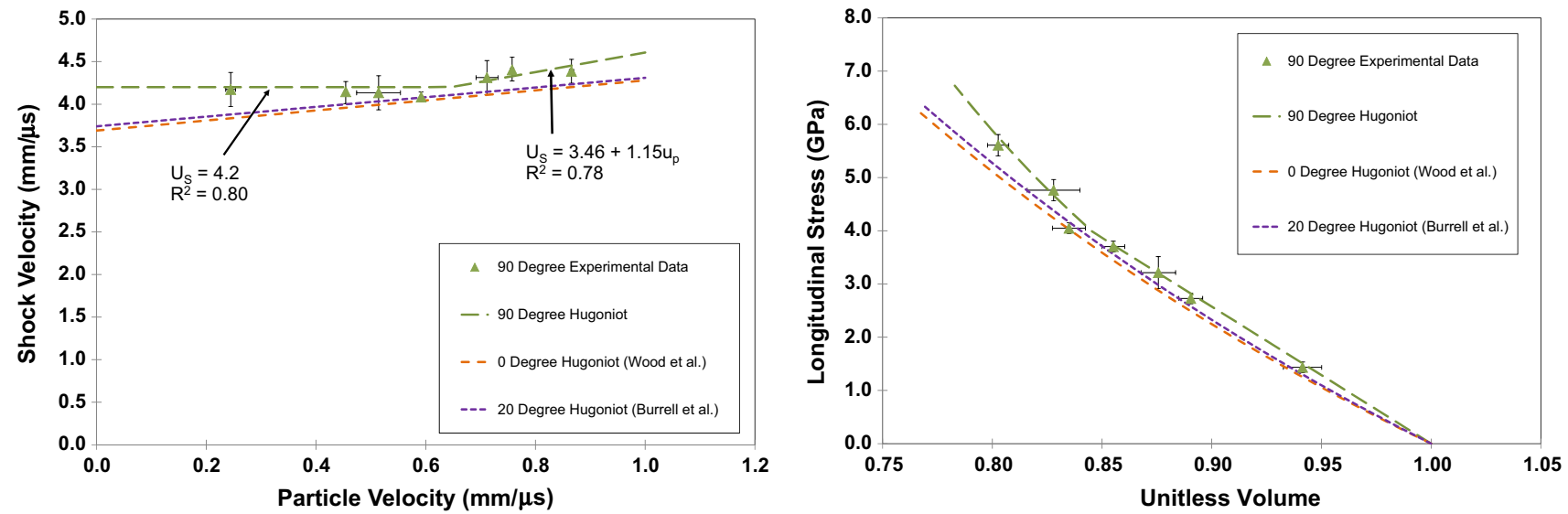

Figure 4 Hugoniot in the shock velocity-particle velocity (left) and pressure-unitless volume (right) plane for the $90^{\circ}$ orientation of the composite TWCP.

Table 6 Hugoniot equations for each orientation of TWCP investigated here as well as the ones investigated by Wood et al. [20] and Burrell et al. [1]

\begin{tabular}{ll}
\hline TWCP orientation & Equation \\
\hline $90^{\circ}$ elastic & $U_{\mathrm{S}}=4.20$; for $u_{\mathrm{p}}$ less than $0.65{\mathrm{~mm} \mu \mathrm{s}^{-1}}^{\circ}$ \\
$90^{\circ}$ plastic & $U_{\mathrm{S}}=3.46+1.15 u_{\mathrm{p}}$; for up between 0.65 and $0.9{\mathrm{~mm} \mu \mathrm{s}^{-1}}^{\circ}$ \\
$45^{\circ}$ & $U_{\mathrm{S}}=3.44+1.12 u_{\mathrm{p}}$ \\
$25^{\circ}$ & $U_{\mathrm{S}}=3.45+0.73 u_{\mathrm{p}}$ \\
$0^{\circ}[20]$ & $U_{\mathrm{S}}=3.69+0.59 u_{\mathrm{p}}$ \\
$20^{\circ}[1]$ & $U_{\mathrm{S}}=3.74+0.57 u_{\mathrm{p}}$ \\
\hline
\end{tabular}

same composite $[1,20]$. Considering first the shock velocity-particle velocity plane, it can be seen that the $90^{\circ}$ orientation has more complex shock behaviour when compared to the more monolithic behaviour exhibited by both the $0^{\circ}$ [20] and $20^{\circ}$ [1] orientations. The Hugoniot for the $90^{\circ}$ TWCP orientation has two distinct regions, an initial response where the elastic wave dominates the material behaviour, and a second region above a particle velocity value of approximately $0.65 \mathrm{~mm} \mathrm{\mu s}^{-1}$ where a more traditional shock response is observed. The equation for both of these regions is shown in Eq. (8). There is little scatter of the experimental data from the line of best fits as shown by the $R^{2}$ values, with a $R^{2}$ value of 0.80 for the initial response and 0.78 for the more standard shock response.

$$
U_{\mathrm{S}}= \begin{cases}4.20, & u_{\mathrm{p}}<0.65 \mathrm{~mm} \mu \mathrm{s}^{-1} \\ 3.46+1.15 u_{\mathrm{p}}, & 0.65<u_{\mathrm{p}}>0.9 \mathrm{~mm} \mu \mathrm{s}^{-1}\end{cases}
$$


Figure 4 also shows the Hugoniot for the $90^{\circ}$ TWCP orientation in the pressure-volume plane. For comparative purposes a unitless volume is employed (primarily for comparison between this composition of TWCP and the one investigated by Burrell et al. Ref. [1], due to the different density). Due to the initial elastic behaviour of the Hugoniot in the $U_{\mathrm{S}}-u_{\mathrm{p}}$ plane for the $90^{\circ}$ orientation, elastic behaviour is also seen in the pressure-volume plane. No substantial deviation was noted between the experimental data and the Hugoniot (Eq. 8) for the $90^{\circ}$ orientations of the TWCP material. As such, no strengthening was seen with the $90^{\circ}$ experimental data, which had previously been seen with the $0^{\circ}$ TWCP orientation investigated by Wood et al. [20].

As mentioned earlier an elastic precursor was observed on the traces in the elastic region, e.g. Fig. 3. Elastic precursors such as this have been seen in carbon fibre composites previously investigated by Hazell et al. [7] and Millett et al. [9], as well as other composites such as the aramid fibre composite investigated by Bordzilovsky et al. [10].

Table 3 shows all of the experimental data which relate to the elastic precursor for the TWCP material with regard to the $90^{\circ}$ orientation. This table includes the velocity of the precursor wave, ascertained from the time between the rise on the front gauge and the start of the precursor on the rear gauge. As the particle velocity of the associated shock wave increased, the velocity of the elastic precursor wave decreased, with the precursor wave disappearing completely

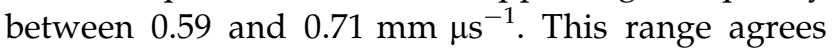
with the elastic to shock wave transition observed in the Hugoniot in the $U_{\mathrm{S}}-u_{\mathrm{p}}$ plane (Fig. 9). This would likely mean that the most probable value that this transition occurs at a particle velocity of $0.65 \mathrm{~mm} \mathrm{ss}^{-1}$; however, due to the lack of experimental data in this region this cannot be definitively proven.

Figure 5 shows a typical trace obtained for the $45^{\circ}$ orientated TWCP sample. Unlike the low velocity $90^{\circ}$ experiments no precursor wave was observed. The other typical trace behaviour was observed such as, sharp rises and plateaus. The experimental data for the $45^{\circ}$ TWCP orientation are shown in Table 4 , with the data plotted graphically in the $U_{\mathrm{S}}-u_{\mathrm{p}}$ and pressure-unitless volume plane in Fig. 6.

The Hugoniot for the TWCP orientated at $45^{\circ}$ is given in Eq. (9). It can be seen that it has a steeper

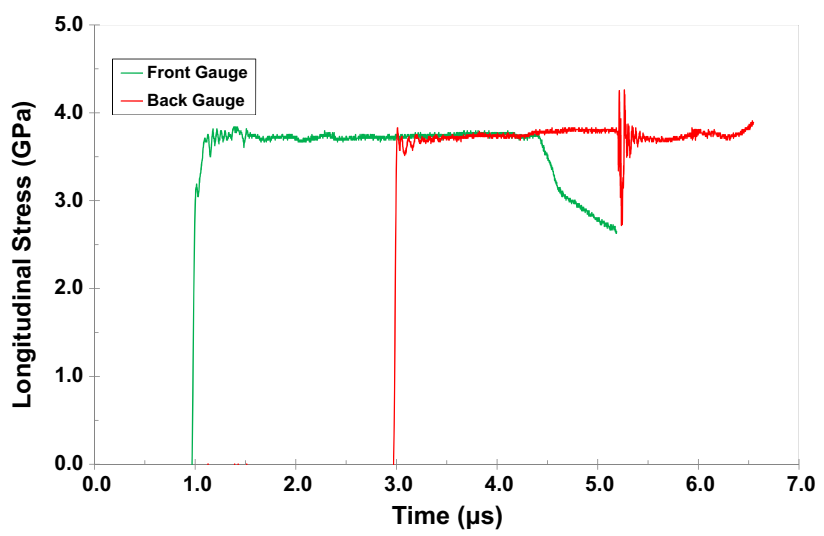

Figure 5 A gauge trace for a $10 \mathrm{~mm} \mathrm{Cu}$ flyer impacting at $700 \mathrm{~m} \mathrm{~s}^{-1}$ for the $45^{\circ} \mathrm{TWCP}$ orientation.

gradient than the Hugoniot observed for the $0^{\circ}$ orientation of TWCP (obtained from Ref. [20]), but the gradient is close to what is observed for the shock portion of the $90^{\circ}$ orientation (as shown in Eq. 8). A small amount of scatter is noted in the data, signified by the $R^{2}$ value of 0.85 .

$U_{\mathrm{S}}=3.44+1.12 u_{\mathrm{p}}$

For the pressure-unitless volume plane no strengthening effects were seen between the Hugoniot and the experimental data. Deviation was observed with the $90^{\circ}$ TWCP orientation as noted between this orientation $\left(45^{\circ}\right)$ and the ones investigated previously $\left(0^{\circ}\right.$ by Wood et at. [20], and the $20^{\circ}$ orientation investigated by Burrell et al. [1]).

Figure 7 shows the manganin gauge trace for the $25^{\circ}$ orientated TWCP sample. As seen with the $45^{\circ}$ orientation no elastic precursor wave were observed, as noted with the $90^{\circ}$ orientated TWCP sample. The trace follows the behaviour noted with the other orientations including sharp rises and plateaus. The experimental data for the $25^{\circ}$ TWCP orientation shown in Table 5.

The data for the $25^{\circ}$ TWCP orientation are plotted in $U_{\mathrm{S}}-u_{\mathrm{p}}$ and pressure-unitless volume plane in Fig. 8, with the calculated Hugoniot in Eq. (10). It should be noted, however, that the scatter on this data is large, compared with the other orientations as demonstrated by the low $R^{2}$ value of 0.49 . This scatter is also observed in the pressure-unitless volume plane as well. While the other orientations agreed well with the Hugoniot in this plane, clear deviation is observed on the highest pressure compared to the Hugoniot. This would normally imply a strengthening effect. However, with the scatter on the 

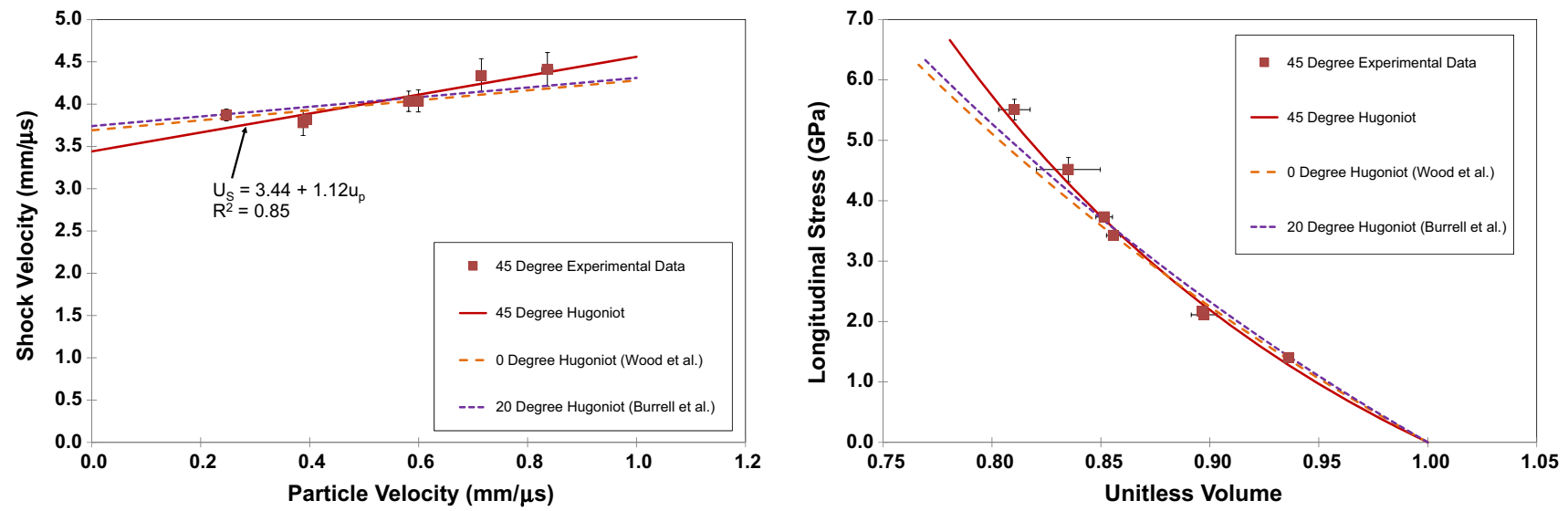

Figure 6 Hugoniot in the $U_{\mathrm{S}}-u_{\mathrm{p}}$ (left) and $\mathrm{P}-\mathrm{V}$ (right) plane for the $45^{\circ}$ orientation of TWCP.

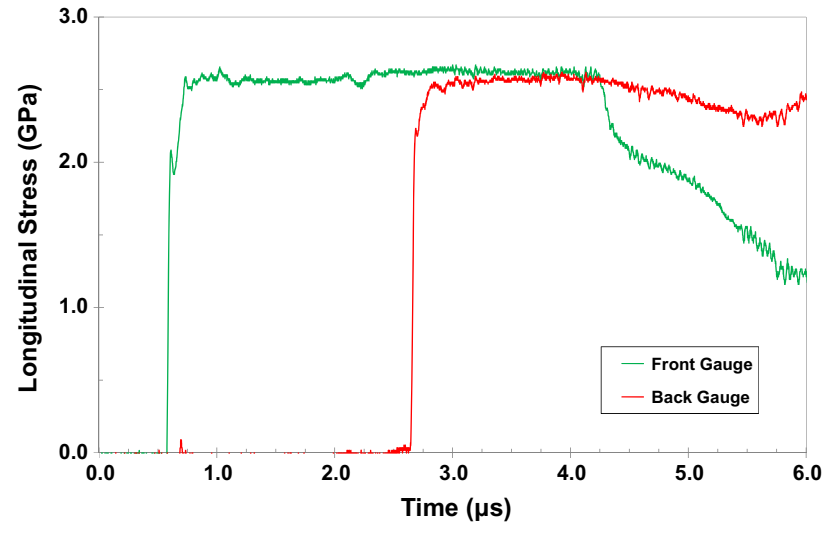

Figure 7 Representative gauge trace for the $25^{\circ}$ TWCP orientation, impacted at $510 \mathrm{~m} \mathrm{~s}^{-1}$ with a $10 \mathrm{~mm} \mathrm{Cu}$ flyer.

experimental data, how statistically realistic this conclusion is, is questionable. The experimental data for this orientation agree closer to the $0^{\circ}$ and $20^{\circ}$ orientations previously investigated by Wood et al. [20] and Burrell et al. [1], respectively. It is reasonable

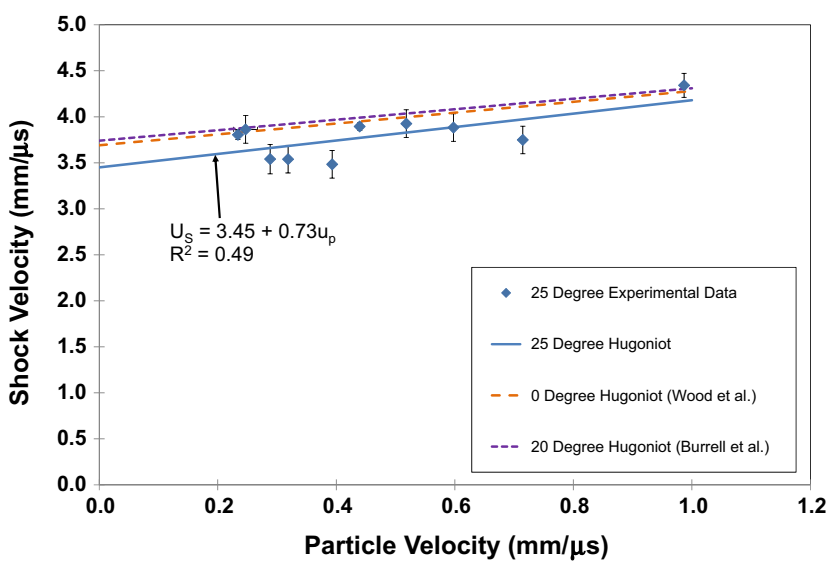

to assume that this scatter is caused by the orientation of the fibre weave within this composite, i.e. the interaction between the fibre weave and matrix material and the shock front, as the other orientation have less scatter associated with them as shown by their higher $R^{2}$ values.

$U_{\mathrm{S}}=3.45+0.73 u_{\mathrm{p}}$

When the Hugoniots of the three investigated orientations $\left(25^{\circ}, 45^{\circ}\right.$ and $90^{\circ}$, with the equations are given collated in Table 6) are contrasted in the $U_{\mathrm{S}}-u_{\mathrm{p}}$ plane (Fig. 9) and pressure-unitless volume plane (Fig. 10), the experimental results are comparable. The exception to this behaviour is the $90^{\circ}$ experimental data, which is due to the elastic precursor dominance at particle velocities below $0.65 \mathrm{~mm} \mathrm{\mu s}^{-1}$. The scatter observed within the experimental data is most notable with the $25^{\circ}$ TWCP orientation. Due to this scatter within the experimental data, convergence of the individual Hugoniot of each orientation

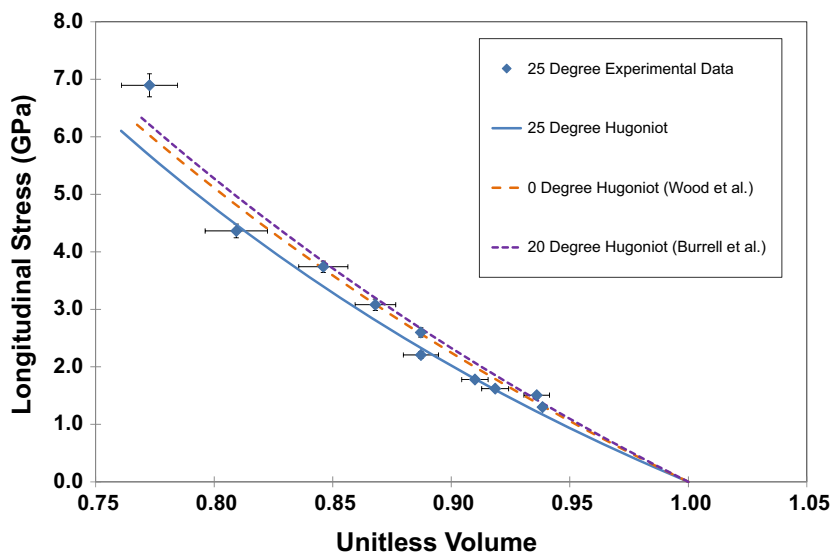

Figure 8 Hugoniot in the $U_{\mathrm{S}}-u_{\mathrm{p}}$ (left) and $\mathrm{P}-\mathrm{V}$ (right) plane for the $25^{\circ}$ orientation for the composite TWCP. 


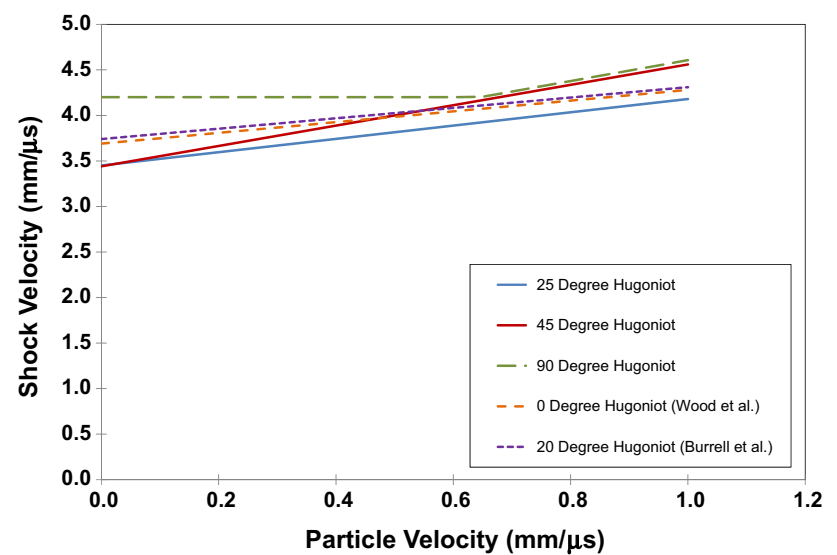

Figure 9 Hugoniot in the $U_{\mathrm{S}}-u_{\mathrm{p}}$ plane, for all of the investigated orientations as well as the literature data for Wood et al. [20] and Burrell et al. [1].

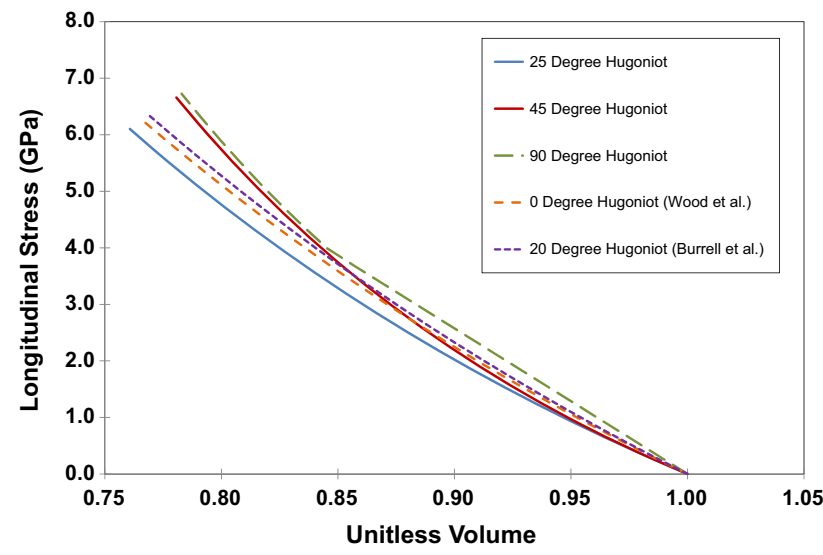

Figure 10 Hugoniot in the pressure-volume plane, for all of the investigated orientations as well as the literature data for Wood et al. [20] and Burrell et al. [1].

did not occur as previously observed with the $0^{\circ}$ TWCP orientation and the phenolic resin matrix material investigated by Wood et al. [20]. With the associated error bars taken into account the majority of the data is broadly similar to the orientations of TWCP previously investigated by Wood et al. [20] and Burrell et al. [1]. Therefore, the Hugoniot for the $0^{\circ}$ TWCP material can be used as an approximation for the shock response of this material. The caveat is that the initial shock response of the $90^{\circ}$ orientation is elastic in nature has to be taken into account. Due to this relative insensitivity of the orientation with regard to the shock response of TWCP; other factors such as ablation, strength etc. can be considered for the materials application.

\section{Conclusion}

The shock response of three different orientations of a tape-wrapped carbon fibre composite with phenolic resin matrix has been investigated. This has been achieved by using the plate impact technique via a single-stage gas gun, with manganin pressure gauges as a diagnostic technique. The orientations of the fibre weave studied here were $25^{\circ}, 45^{\circ}$ and $90^{\circ}$ with respect to the impact face. These data have been contrasted with the previously obtained data for other TWCP orientations by Wood et al. [20] and Burrell et al. [1], who used a different composition within their TWCP material, as well as fibre weave orientation. When all of the orientations are compared to one another, little difference was observed with the exception of the $90^{\circ}$ TWCP orientation. This was due to elastic behaviour below a particle velocity value of $0.65 \mathrm{~mm} \mathrm{\mu s}^{-1}$. Also noted within the data set was an amount of scatter present, especially with the $25^{\circ}$ TWCP orientation. This is most likely induced by the differing constituents within the composite, leading to a more dispersed and complex wave profile. This therefore means that other properties such as strength, ablation etc. may be considered for the materials application.

\section{Acknowledgements}

The authors would like to acknowledge the support of the Institute of Shock Physics and AWE. LockheedMartin Insys is also thanked for supplying the experimental samples. In addition, we would also like to thank Mr. Andrew Roberts for technical help in carrying out the experiments. British Crown Copyright MOD/2018.

Open Access This article is distributed under the terms of the Creative Commons Attribution 4.0 International License (http://creativecommons.org/ licenses/by/4.0/), which permits unrestricted use, distribution, and reproduction in any medium, provided you give appropriate credit to the original author(s) and the source, provide a link to the Creative Commons license, and indicate if changes were made. 


\section{References}

[1] Burrell RH, Barnes NR, Keightley PT et al (2009) The response of a carbon fibre-phenolic resin composite to onedimensional shock loading. In: 16th APS topical conference on shock compression of condensed matter

[2] Windhorst T, Blount G (1997) Carbon-carbon composites: a summary of recent developments and applications. Mater Des 18:11-15. https://doi.org/10.1016/S02613069(97)00024-1

[3] Wicklein M, Ryan S, White DM, Clegg RA (2008) Hypervelocity impact on CFRP: testing, material modelling, and numerical simulation. Int J Impact Eng 35:1861-1869. https://doi.org/10.1016/j.ijimpeng.2008.07.015

[4] Millett JCF, Bourne NK, Deas D (2005) The equation of state of two alumina-filled epoxy resins. J Phys D Appl Phys 38:930-934. https://doi.org/10.1088/0022-3727/38/6/023

[5] Dattelbaum DM, Coe JD, Rigg PA et al (2014) Shockwave response of two carbon fiber-polymer composites to 50 GPa. J Appl Phys 116:194308. https://doi.org/10.1063/1. 4898313

[6] Oved Y, Luttwak GE, Rosenberg Z (1978) Shock wave propagation in layered composites. J Compos Mater. https:// doi.org/10.1177/002199837801200107

[7] Hazell PJ, Stennett C, Cooper G (2009) The effect of specimen thickness on the shock propagation along the in-fibre direction of an aerospace-grade CFRP laminate. Compos Part A Appl Sci Manuf 40:204-209. https://doi.org/10.1016/ j.compositesa.2008.11.002

[8] Tsai L, Prakash V, Rajendran AM, Dandekar DP (2007) Structure of shock waves in glass fiber reinforced polymer matrix composites. Appl Phys Lett 90:061909. https://doi. org/10.1063/1.2435340

[9] Millett JCF, Bourne NK, Meziere YJE et al (2007) The effect of orientation on the shock response of a carbon fibre-epoxy composite. Compos Sci Technol 67:3253-3260. https://doi. org/10.1016/j.compscitech.2007.03.034

[10] Bordzilovsky SA, Karakhanov SM, Merzhievsky LA (1998) Shock response of a unidirectional composite at various orientation of fibers. In: 10th APS topical conference on shock compression of condensed matter, pp 545-548

[11] Millett JCF, Meziere YJE, Bourne NK (2007) The response to shock loading of a glass-fibre-epoxy composite: effects of fibre orientation to the loading axis. J Phys D Appl Phys 40:5358-5365. https://doi.org/10.1088/0022-3727/40/17/ 052
[12] Meyers MA (1994) Elastic waves. In: Meyers MA (ed) Dynamic behavior of materials, p 55. Wiley, New York. https://doi.org/10.1007/s11340-012-9598-0

[13] Barker LM, Hollenbach RE (1970) Shock-wave studies of PMMA, fused silica, and sapphire. J Appl Phys 41:4208-4226. https://doi.org/10.1063/1.1658439

[14] Carter WJ, Marsh SP (1995) Hugoniot equation of state of polymers

[15] Shepherd CJ, Appleby-Thomas GJ, Wilgeroth JM et al (2011) On the response of ballistic soap to one-dimensional shock loading. Int J Impact Eng 38:981-988. https://oi.org/ 10.1016/j.ijimpeng.2011.07.003

[16] Wood DC, Hazell PJ, Appleby-Thomas GJ, Barnes NR (2011) Shock behaviour of a phenolic resin. J Mater Sci 46:5991-5999. https://doi.org/10.1007/s10853-011-5560-2

[17] Davison L (2008) Fundamentals of shock wave propagation in solids. Springer, Berlin

[18] Appleby-Thomas GJ, Hazell PJ (2012) The impact of structural composite materials. Part 2: hypervelocity impact and shock. J Strain Anal Eng Des 47:406-418. https://doi. org/10.1177/0309324712448299

[19] Willows M, Tsembelis K, Proud WG, Church PD (2007) Shock characterisation of a carbon fibre composite. In: 15th APS topical conference on shock compression of condensed matter. Poster presentation only

[20] Wood DC, Appleby-Thomas GJ, Hazell PJ, Barnes NR (2012) Shock propagation in a tape wrapped carbon fibre composite. Compos Part A Appl Sci Manuf 43:1550-1560. https://doi.org/10.1016/j.compositesa.2012.04.013

[21] Marsh SP (1980) LASL shock hugoniot data. Los Alamos Ser Dyn Mater Prop, p 150. ISBN 0-520-04008-2

[22] Rosenberg Z, Yaziv D, Partom Y (1980) Calibration of foillike manganin gauges in planar shock wave experiments. J Appl Phys 51:3702-3705. https://doi.org/10.1063/1. 328155

[23] Appleby-Thomas GJ, Hazell PJ, Stennett C (2009) The variation in lateral and longitudinal stress gauge response within an RTM 6 epoxy resin under one-dimensional shock loading. J Mater Sci 44:6187-6198. https://doi.org/10.1007/ s10853-009-3859-Z

[24] Hazell PJ, Appleby-Thomas GJ, Trinquant X, Chapman DJ (2011) In-fiber shock propagation in Dyneema ${ }^{\circledR}$. J Appl Phys 110:043504. https://doi.org/10.1063/1.3622294 